\title{
Status and Prospects of Agricultural Information Technology*
}

\author{
Liu Ji-fang ${ }^{1,2, a}$, Wu Jian-zhai ${ }^{1,2, b}$, Zhang Jian-hua ${ }^{1,2}$, Liu Jia-jia ${ }^{1,2}$ \\ ${ }^{1}$ Agricultural Information Institute, China Academy of Agricultural Sciences, Beijing, 100081, China; \\ ${ }^{2}$ Key Laboratory of Agri-information Service Technology, Ministry of Agriculture, Beijing, 100081, China \\ aliujifang@caas.cn, bujianzhai@caas.cn
}

\begin{abstract}
This paper thoroughly analyzes the development levels and problems of agricultural information technology, puts forward the practical needs of modern agricultural development and the prospects of information technology. It describes the development trends of agricultural information technology and concludes key technologies and R\&D tasks to be carried out in the future.
\end{abstract}

Keywords-Agriculture; Information Technology; Current Situation; Prospect.

\section{INTRODUCTION}

Over the past few decades, the growing fusion of information technology (IT) and agriculture have resulted in rapid development of agricultural information technology (AIT). IT has been applied in every agricultural stage and played essential roles in spreading knowledge, orienting information and making scientific decisions. A new pattern is emerging in China's agricultural development. Agricultural modernization turns into an important theme of the economy in the new era, which requires thorough integration of agriculture and information. AIT will forcefully support the development of modern agriculture. Information technologies will help agricultural innovation, playing an important role in modern agriculture development.

\section{Status of SCIENCE AND TECHNOLOGy DEVELOPMENT}

In the field of agricultural information technology, new technologies such as Internet of Things (IOT) and cloud computing have been applied in farming resources, plant and animal breeding, crop cultivation, livestock and poultry feeding, soil fertilizer and plant protection. For example, the field of production witnessed the application of technologyintensive machinery which has global positioning and intelligence technologies. Wireless sensor network and automatic control technology have updated water conveyance and irrigation facilities. Intelligent control technology and equipment have realized fully automatic farming and early detection of diseases.

In the field of agricultural information analysis, basic theory system has been improved and a monograph named "Agricultural Information Analytics" had been published. Methods and technologies identifying risk factors in agricultural production and market have been developed. Technological systems have been established for

"This work was supported by the National Natural Science Foundation of China (41201599) quantitatively evaluating the risks in agricultural industry chain, price transmission and price forecasting of agricultural products, simulation of food security scenarios. Breakthroughs have been made as to service technologies, such as initiative push, embedded service and addressable broadcasting. The technologies on collecting, transmitting and processing information of agricultural market have been innovated and advanced information acquisition tools have been developed. Large agricultural information analysis system represented by agricultural monitoring and early warning system and economic map have been promoted and applied throughout the country.

In the field of agricultural information management, digital information environment and demands for agricultural science and technology have undergone profound transformation. The information resources and digital environment are experiencing revolutionary changes. Digitalization and open access gradually become the trend of technology information resources. Knowledge creation, organization, dissemination and utilization are forming new patterns. With the support of information technology, technological information acquisition and services are professional, user-oriented and knowledgebased, growing rapidly.

In the field of agricultural remote sensing, wireless sensor network has been applied in monitoring environmental ecology, and rural environment and farmland parameters. A worldwide remote sensing database with multiple bands, angles and high time resolution, a multi-source Earth observation satellite system have been established and widely used in simulations of land evaluation, soil interpretation and nutrients management. National remote sensing systems have been working stably to monitor crops, drought and flood disasters in our country. Agricultural remote sensing technologies develop towards quantization and product-based. The applied researches have been carried out in the productivity evaluation, disaster monitoring and ecological degradation. Remote sensing experiments and researches have been carried out on the satellite-machine-land platform.

While AIT has been developed rapidly, there are still some deficiencies.(1) Short of data standardization and integration: poor consistency of standards for agricultural data, obscure indexical connotation and ambiguous data attributes; some questions expect further researches, such as how to integrate data from different observation systems, how to integrate directly and indirectly observed data, how to integrate observation data and simulated results of the models, and how 
to deduct data fusion degree. (2)The originality and sensitivity of technical methods need to be strengthened: information technology and information analysis of agriculture lagged behind other industries. The originality of AIT needs to be strengthened on the basis of understanding the law of agricultural development. Meanwhile, the sensitivity to new technologies and application of cutting-edge information technology shall be enhanced. (3)High-end information analysis system needs improvement: since China is a large agricultural country, present model cannot meet the needs of the agricultural products markets and decision-making process. A comprehensive and systematic platform shall be built to support decision-making process. (4)Low utilization of agricultural technology and information resources: the disordered and sporadic information made it difficult to access and utilize. The restricted utilization level hinders innovation of agricultural science and technology.

\section{The DEVELOPMENT TREND OF SCIENCE AND TECHNOLOGY}

In developed countries, agricultural normalization started after agricultural industrialization and mechanization, aiming at figuring out how to improve labor productivity, resource utilization, economic benefits, environmental protection and sustainable development. With the rapid development of computers, artificial intelligence, network and multimedia information technology, AIT will become intelligent, standardized, integrated and networked. It will become a key production element which is integrated into every aspect of agricultural industry.

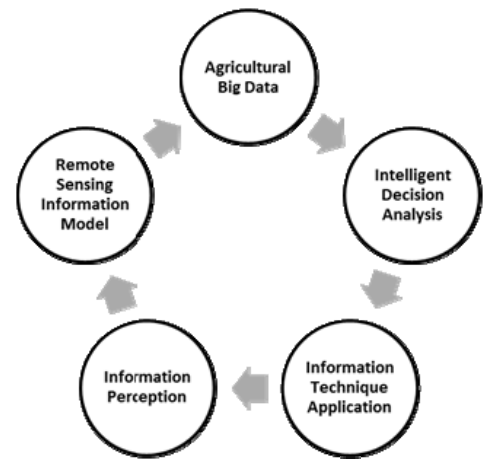

Fig.1. Direction of AIT development

\section{A. Rapid development of agricultural big data, standardization of knowledge resources and sharing technologies}

In the future, agricultural big data will become a hotspot. Data acquisition will rely more on the IOT, mobile terminals and remote sensing technology. Instant messages of production, market and consumption data are collected immediately. Data mining technology will be applied to analyze instant data, historical data and predicted data before forecasting and early warning are made, which will play a greater role in providing decisive basis for the government's macroeconomic control. Agricultural ontology service will provide a modern framework to define and manage agricultural terminologies. This framework will support the standardization of agricultural information resources and information sharing. Its combination with web-based search tools will benefit resource retrieval. In addition to providing query results to specific users, it presents related resources that the user might be interested in.

\section{B. The applications of intelligent technology in agricultural analysis and decision-making system are increasing}

In the future, agricultural control, management and service will become intelligent. Cutting-edge technologies, such as bionic intelligence, data mining, and complex systems, especially the unprecedented breakthroughs in evolutionary algorithm, immune algorithm and swarm intelligence, will contribute theories for constructing an agricultural analysis and decision system to realize computer-assisted decisions, which are based on intelligence agent and knowledge ontology in Internet environment. As to means of realizing these objectives, ambient intelligent mobile terminals will develop rapidly with the popularity of mobile Internet technology. They will be applied to all elements throughout the process, and will cover the entire system.

\section{AIT application and development become more and more cooperative, comprehensive and omni-directional}

In the future, AIT application will no longer be limited to a procedure or a production process. The scope of application and development will be expanded from research to education, from production to management, from macro scale to Micro scale, from management to technology. It will involve almost all agricultural issues. Comprehensive application of technologies and disciplines enables AIT to greatly improve agricultural production process and modes, and promote the level of agricultural modernization. The all-dimensional, comprehensive and cooperative AIT application and development of AIT are of great significance for accelerating the transformation of agricultural production mode and cracking development and application challenges.

\section{The application of new technologies and new materials in information perception will be highlighted}

One of the directions of AIT development is biological information perception with sensors made of biomaterials. Traditional sensing receptors are completely made of nonliving materials. To the contrary, biosensors are made of or have biomaterials. Biomaterial sensor is featured with better specificity, high sensitivity, rapid analysis, high accuracy and low cost. It will become a exploring hotspot and research frontier at home and abroad. The growing processes of crops are closely related to their biological information of themselves, as well as external inductions. The acquisition and determination of biological information are essential to cracking the growth codes of the crops.

\section{E. Remote sensing information model is a research frontier in the future}

A remote sensing information model is crucial to the application of sensing technologies. It can be used to calculate and back calculate agricultural parameters that are valuable in 
practices. Over the past few years, people have developed a number of remote sensing information models, such as greenness index model, crop yield estimation model, drought index model, temperature index model, field evapotranspiration estimation model and soil moisture monitoring models. However, they are far from enough to meet the needs of remote sensing. Therefore, the development of new models is still the frontier of remote sensing research. More efforts shall be invested to establish and test integrated remote sensing models for crop, resources and grassland vegetation.

\section{Key TECHNOLOGIES EXPECTING BREAKTHROUGHS}

As an important part of modern agricultural technology system, AIT propels the growth of agricultural productivity and rural economic and social development. Any AIT research shall make full use of all kinds of information inside or outside the agricultural system, not matter it is dynamic or static, quantitative or qualitative, accurate or ambiguous. In this way, energy conversion and materials circulation processes in agricultural system will be effectively.

\begin{tabular}{|c|c|c|c|}
\hline Perception \\
$\begin{array}{c}\text { Physiological } \\
\text { Activity }\end{array}$ & Data & Pocess & Sisualization \\
Information
\end{tabular}

Fig.2. Technical ideas

Therefore, from the perspective of long-term development, researches should focus on following four key technologies:

\section{A. Data acquisition and mining technology}

Based on the IOT and targeted at growing and physiological phenotypes of crops of different strains, with different cultivation patterns and moisture treatments, comparison and analysis are made to distinguish the differences, advantages and disadvantages of manual measurement equipment, sensor-based equipment, network transmission equipment and other parameter measurement equipment. The achievements will be used to develop fast, non-destructive and accurate acquisition of phenotypic parameters with sensors and network transmission technologies. Modern IT and NT will be applied in the fundamental and theoretical researches on biosensors, with the help of material science, biological science, crop science and information science. Researches will be carried out on biomaterial sensing technologies and biosensors manufacturing. Portable data acquisition devices will be developed for real-time acquisition of crop grown area, crop growth and plantation structure in the exemplary bases. By focusing on the construction of five categories of reference data (agricultural natural resources, agricultural production, agricultural market, agricultural management, information science and technology) and data application services, more efforts will be put to develop a reference data system and technology of collecting reference data. Accurate data analysis model and technological researches on correlation analysis, data mining, knowledge discovery will be enhanced to establish a technology system for reference data of modern agriculture.

\section{B. Agriculture intelligent simulation technology}

In the field of agricultural production, researches will focus on how to integrate intelligently perceived crop environment data and parameter database of the crop simulation model, how to design intelligent simulations of crop growth, environment and management measures, how to construct crop - environment simulation model, how to quickly reconstruct 3D morphological model based on cloud data of 3D points, how to acquire structure parameters of the crops, how to automatically acquire morphological characteristics (crop branch diameter, crown diameter, total leaves, breach length ratio) in complex environment, and how to construct parametric model of 3D morphological structures. Following technologies will also be studied and promoted in representative regions: smart simulation of crop growthenvironment interaction, key technologies of smart management of crop growth process, construction of modeloriented universal control module, smart control robots with independent intellectual property rights, smart equipment system that will replace manpower. In the field of market circulation, researches will focus on early-warning algorithm based on data-intensive and data-driven conditions, technologies on matching the variety, supply, demand, price, time and space of the agricultural products, and dynamic multi-dimensional visualization simulation of market circulation. Since agricultural products are in a perfectly competitive market, studies should center around buyers, sellers and the "invisible hand" of the market. Progresses should be seen in behavior simulation technology in agricultural macro system, as well as the modeling and simulation of transaction behaviors in agricultural market. According to the smart forecasting and early warning of the agricultural products market, studies will be carried out to intelligently visualize market regulation simulations in a digital manner. Therefore, the agricultural market regulation decisions will be optimized.

\section{Agricultural cloud services technology}

Through studies on integrating cloud computing resources in heterogeneous or offsite environments, IT resources of cloud computing and cloud storage in said environments will be integrated into a unified pool to meet the needs of scalability, interoperability and multiple centers. Scheduling of resources in operation systems with different granularities and dimensions will be studied to realize nationwide promotion of suitable operation systems for agricultural studies. Because different operation systems vary in computing granularities and technical dimensions, cloud computing resources will be provided in a differentiated manner. Unified interfacing of cloud computing resources will researched to provide applications of agricultural technologies with cloud computing resources access, cloud storage resource access, cloud database access, message scheduling access, unified security access, to form a unified resource access for different technical demands and standard specification, to provide access services to developers of applications and platforms. At the same time, the core tasks will be improving cloud service platforms for network management, information dissemination and application services. By targeting at construction of big data for agricultural researches, 
agricultural science data center and high-performance cloud computing platform, focusing on animal and plant breeding, quality safety of agricultural products and agricultural science and technology management, studies of key technologies ( information acquisition, data processing, knowledge discovery, smart push) are carried out to satisfy the increasing demands of agricultural science and technology personnel for big data and cloud computing technologies. To be specific, the researches include data processing technology based on main memory database, indexing model for security data with complex structures, fusion model for large-scale and multisource heterogeneous security data, data mining model, visualization analysis of massive security data.

\section{Livestock Perception and Smart Control Technology}

Livestock environment perception will be studied. According to the needs of collecting environment data, data acquisition and transmission of meteorological sensors (including temperature, humidity, light intensity, rainfall, etc.), gas sensors (O2, CO2, CO, NH3, CH4)will be studied for designing equipment interfaces available for multiple sensors and for collecting data of livestock environment. By studying intelligent control of livestock environment and comparing a variety of control methods, intelligent control will be ultimately realized. The information collected by the sensors will be fused. Artificial intelligence control will be used to realize nonlinear control. Thus, these measures will ensure the best environment for livestock and poultry. Individual livestock and poultry will be monitored from time to time with audio, video, infrared and other modern information technologies. Their growth information (such as weight, body temperature, respiration, heart vessel, walking, etc.) will be measured in time. With the help of artificial intelligence and image analysis, early-warning model for epidemic diseases will be studied to forecast and control livestock and avian diseases. An information management platform for livestock will be constructed to fuse, process and analyze the environmental information, gas information and individual information from pastures. Then, intelligent operation terminals will be applied to monitor, control and make scientific decisions before, during and after the production. At last, human, facility and materials in the pastures will be integrated.

\section{RESEARCH AND DEVELOPMENT FOCUSES}

\section{A. Research, development and application of IOT-based digital simulation and monitoring technologies}

At present, agricultural production management still depends on experiences and weather. There is no fundamental change. As experienced farmers getting old, a new concern is about how to use modern information technology, agricultural science and technology achievements and growing agricultural data to effectively manage crop production, forecast and deal with crop growth bottlenecks, and provide better environment for crop growth. The research is of great significance on improving utilization of agricultural resources, and ensuring national food security.
In the future, studies should use big data to develop data mining equipment, image recognition system and visualization systems, smartly acquiring and mining useful information from network, video surveillance, statistics, remote sensing information and weather information. Crop growth mechanism should be used to explore growth model of main crops and to develop technologies that can simulate crop growth. Experiments and simulation will facilitate converting observed information into environmental messages that directly impact crop growth. Construct an index system of crop growth and establish the methods and mechanism to monitor crop growth. Analyze and monitor crop growing, drought, pests, nutrient levels, yield structure and market prices. Integrate information acquisition, growth simulation, environmental modeling and monitoring data. Develop the system for monitoring and controlling crop production and management. Realize the automation and intelligence of production monitoring and control.

\section{B. Crop simulation model}

By studying major food crops, fruit trees and cash crops, analyzing domestic and abroad researches on crop models, adopting simulation models, networking and artificial intelligence technology, simulation of virtual environment of main crops will support decisions on high yielding fertilization measures and soil fertility improvement, and provide technological and theoretical basis for smart management of agricultural production in China.

Future studies should analyze the mechanisms that crop varieties, weather conditions, soil level, human management practices and pests impact the growth of food crop and cash crop and explore their coupling effects. The sensors' diversities will be considered in developing corresponding data acquisition, transmission and pretreatment technologies. Researches will be carried out on optimizing the distributed topology of multivariate devices to collect crop growth and environmental information. An Agent-based intelligentcooperation model of crop grow will be constructed based on the model of main factors that impacts crop growth, with the adoption of networking technologies and agent technology.

\section{Research and development of livestock perception and smart control equipment}

At present, animal husbandry in China is transiting from the traditional stage to modern one. In animal husbandry, modern science and technology will be applied in every aspect of management and operation. Perception technology will be applied in every step of breeding. Smart control and operation devices will be developed. Technologies and equipment supports will be provided for modernized and informationbased management.

Aiming at the difficulties of real-time perception of breeding environment and individual information, IOT technology and machine vision technology should be used as the basis to thoroughly analyze the characteristics of meteorological and gas perception, to study information acquisition and fusion of multiple sensors, to explore how to express vital signs of individual livestock, and to construct a 
system to perceive and visualize environmental and ontological information in animal husbandry. Breakthroughs are expected with respect to remote nonlinear smart control technologies by integrating information collected by sensors, livestock breeding repository and artificial intelligence technology; Design a multi-layer integrated electronic circuit which is small, portable and smart. Develop monitoring equipment and smart control devices for all aspects of animal husbandry. Construct a model for early recognition of epidemic diseases based on machine vision technology and behavioral expressions of the animals.

\section{Research and construction of a large scale system for smart warning of agricultural information}

Due to the complexity of agricultural systems, warning systems with better coverage and stability have been developed by FAO, the United States and EU, which is a great effort to promote national or regional sustainable development of the agriculture and protect agricultural benefits of the region or country. Currently, agricultural monitoring and early-warning systems belong to different sectors and lack of correlation, authority and applicability. The research and construction of a large-scale agricultural system is an urgent issue to be solved when agriculture in China develops into a new stage. It is necessary for stable development of agriculture.

Researches shall focus on data density, data aggregation, intelligent analysis, quick application of high-value data. Efforts shall be made to construct a macro system and service platform for unified, comprehensive analysis of agricultural information. Conduct researches on model and data synchronism. Coordinate and deploy smart agent services. Realize unified management and scheduling of application systems and early warning analysis models. Conduct researches on intelligent warning based on multi-agent technology and develop relevant components. Explore intelligent generation of early warning and response plans. Conduct researches on visualizing early warning plan and main results. Develop components for dynamic and multidimensional visualization.

\section{E. Researches on deep aggregation of digital resources and associated technologies.}

With the development and application of Internet technologies, acute problems emerge in information explosion, complex semantic relationship between objects, dynamic changes of data, communicating difficulties between heterogeneous resources. The network is filled with disordered and fragment information. Quick access to useful information needs processing and organizing clues in the sea of organization. These problems shall be solved. The vital tasks of agricultural management include improving the utilization and reuse rates of resources, promoting the development of knowledge discovery and knowledge services, and providing a better environment for sharing and communicating knowledge.

Conduct researches on new technologies and new methods of documents digitization, discovery, acquisition and organization of open Internet resources. Make breakthroughs in key technologies of acquiring, processing and indexing knowledge. Collect, integrate and associate agricultural technology information. Construct large-scale digital knowledge warehouse rich in semantic associations. Promote the organization and reveal of multi-granularity information about agricultural science and technology. Realize deep aggregation and computable integration of resources.

\section{F. Research on knowledge service in large data environment}

With the development of the Internet and other modern information technology, agricultural science and technology innovation and research environment have changed dramatically. Scientific users have new information needs: digitization, networking, collaboration, specialization and sharing. These changes put forward a serious challenge to traditional service technology and mode. An issue that information service agencies are facing with is how to provide tailored and specialized services to different research agencies. In the big data environment, service innovation is no longer theoretically or strategically difficult. Instead, it becomes a problem of knowledge aggregation and services convergence, which are user-oriented.

\section{SUMMARY}

In the agricultural filed, key knowledge service technologies such as automatic information acquisition, intelligent processing of large-scale data, knowledge organization and integration, will be applied, integrated and optimized to construct a multi-dimensional knowledge service system and supporting technology platform based on providing one-stop integration services to scientific research personnel, innovated by providing profound knowledge services to different research institutions, research teams and professional fields, and expanded by supporting online academic communication and research collaboration. By collecting and integrating user-oriented knowledge resources, researching and applying knowledge connection, knowledge navigation, intelligent retrieval and other knowledge service technologies, the users' demands for tailored knowledge services will be satisfied. The service innovation should gradually develop into a mode focusing on user needs, supported by information technologies and oriented by mining the value of knowledge services, which takes network of relationship (super network) as its macro perspective and provides comprehensive support for agricultural researches.

\section{References}

[1] Wang Wensheng,Research Progress and Development Trend of Agricultural Information Technology in German;Agricultural Outlook, Sep., 2011:48-51.

[2] Zeng Xiaohong, Wang Qiang.An Overview of the Development of Domestic and International Agricultural Information Technology and Network; Chinese Agricultural Science Bulletin, 2011,27(8): 468-473.

[3] 5Li Daoliang,Zhang Liwei.Agricultural Information Technology and Modern Agriculture Development [J].China Rural Science \& Technology, 2007 (12): 30-31.

[4] Shen Ying.Domestic and International Trends of Agricultural Information Development [J].World Agriculture, 2002(1):43-45.

[5] Zhou Guomin.Status and Prospects of Korean agricultural information technology [J].World Agriculture, 2002, 10: 31-33. 
[6] Yang Wenping,Hu Xiqiao.On agricultural information technology and its development trend [J].Jilin Agricultural Sciences, 2010,35 (1): 61-64.

[7] Zhang Xiao. EU's New Strategies on Agricultural Information Technology Research and Development and Implications for ChinaWorld Agriculture, 2013,9: 14-18.
[8] Sun Shimin,Zhou Yanping,Jing Fengshui,Chen Huiying,Li Guangti.On agricultural information technology and agricultural modernization. Research of Agricultural Modernization, 1999,20 (4): 211-214. 